\title{
Identification of Immune Cell Infiltration and Effective Diagnostic Biomarkers in the Lesion of Endometriosis Based on Multi-Bioinformatic Analysis
}

ke Zhang

Third Affiliated Hospital of Southern Medical University

Lihao Zou

Third Affiliated Hospital of Southern Medical University

Xiao Xie

Third Affiliated Hospital of Southern Medical University

Suiqun Guo ( $\sim$ guosq2005@126.com )

Third Affiliated Hospital of Southern Medical University

\section{Research Article}

Keywords: endometriosis, ectopic lesion, immune infiltration, diagnostic biomarker, bioinformatic analysis

Posted Date: February 1st, 2022

DOI: https://doi.org/10.21203/rs.3.rs-1305082/v1

License: (c) (i) This work is licensed under a Creative Commons Attribution 4.0 International License.

Read Full License 


\section{Abstract}

Background: In recent years, it is proved that the immune system contributes a lot to the pathogenesis and progression of endometriosis (EM). Identifying the signature of immune cell infiltration, the immune related diagnostic biomarkers, and even the potential therapeutic targets in endometriosis patients will aid the early diagnosis and treatment of EM.

Methods: Firstly, the microarray expression dataset was downloaded from the gene expression omnibus (GEO) database. Subsequently, the signature of immune cell infiltration of endometrium tissues was calculated through the $x$ Cell algorithm. The hub module most relevant to immune cells was selected through the weighted gene co-expression network (WGCNA). The differentially expressed genes (DEGs) were identified by the limma package of R. The immune-related hub genes were further selected. Finally, the validation and clinical characteristics of hub genes were performed.

Results: In our study, it was showed that macrophages and neutrophils were the main infiltrating immune cells in the endometrium tissue and 4 hub genes related to immune infiltration in ectopic lesions of EM were identified through multi-bioinformatic analysis. The expression of those hub genes (TNFSF13B, IL7R, CSF1R, LEP) in other validated datasets was consistent with our results. Each hub gene had significantly correlation with most of immune cells, and the area under the ROC curves (AUC) of those hub genes for disease diagnosis were all higher than 0.8. We also find that those hub genes were connectively concerned with the common complication infertility of EM.

Conclusions: It is proved that the dysfunction of the immune system is connectively associated with the ectopic lesions of EM. And the immune related biomarkers (TNFSF13B, IL7R, CSF1R, LEP) may be helpful in the development of the pathogenesis, diagnosis and even the potential therapeutic targets of EM.

\section{Background}

Endometriosis is defined as the presence of functional endometrial glands and stroma outside the uterine cavity with the most common locations for the ectopic endometria (EC) being the ovaries. About 6 to $10 \%$ of women of reproductive age have been suffering endometriosis with a series of annoying symptoms, including dysmenorrhea, chronic pelvic pain, and infertility $(1,2)$. Despite decades of research, the etiology and pathogenesis of endometriosis remain unclear. Multiple theories exist regarding its etiology, including the retrograde menstruation theory, hormonal conditions, gene profiles, immune disturbances (3). Currently, laparoscopy is the "gold standard" to diagnose and stage endometriosis, while the mean latency between the onset of symptoms to definitive (surgical) diagnosis is 6.7 years (4). Therefore, it is urgent for us to explore the molecular mechanisms and novel diagnostic biomarkers underlying endometriosis.

Mounting research suggests that disturbed local and systemic immune systems are involved in the poor clearance and persistence of ectopic endometria of EM patients $(1,5,6)$. The immune environment within the ectopic lesions of women with endometriosis is largely different from that within normal endometria, 
such as abnormalities in various cell types increased levels of activated peritoneal macrophages, abnormal activation of $\mathrm{T}$ and $\mathrm{B}$ lymphatic cells, various proinflammatory and regulatory cytokines (7-9). Abnormal activated immune cells at and around endometriotic lesion sites can induce the release of a range of proinflammatory mediators and cytokines, which have been shown to promote the persistence of lesions $(6,10)$. Disturbed local and systemic immune systems in women with endometriosis have been also shown to increase rates of implantation failure and contribute to infertility in women with endometriosis (11).

Over the last decades, with the advancement and availability of high-throughput technologies, microarrays and RNA sequencing, large-scale transcriptome data were produced, which can provide a comprehensive understanding of the molecular mechanisms underlying concerned diseases $(12,13)$. The novel gene signature method xCell (http://xCell.ucsf. edu/) was used to investigate 7 types of infiltration immune cells using extensively in silico analyses based on gene expression data (14). The weighted gene co-expression network analysis (WGCNA) can screen gene modules closely related to concerned diseases by analyzing the correlation between genomic and clinical information, thus providing a basis for further research (15). The Immunology Database and Analysis Portal (ImmPort) repository, applied in the validation of the methods used in the original studies, leveraging studies for meta-analysis, or generating new hypotheses, is an online database created for better immunology research in the future $(16,17)$. In this study, we attempt to analyze the novel, and for the first time, biomarkers related to immune cell infiltration and explore in ectopic endometria of EM patients and explore its clinical characteristic using multi-bioinformatic analysis.

\section{Methods}

2.1 Data collecting. The gene expression microarrays of GSE141549 downloaded from the GEO database, was based on GPL10558(Illumina HumanHT-12 V4.0 expression beadchip) and GPL13376(Illumina HumanWG-6 v2.0 expression beadchip) platforms and has been initially corrected to move the batch effects and combined (18). It included 408 samples obtained from healthy and patient endometrium, peritoneum, and patient endometriosis lesions. In this study, all enrolled samples we further analyzed were obtained from ectopic lesions (EC) of endometriosis patients and normal endometria (NE) of healthy peoples without hormonal medication, including $102 \mathrm{EC}$ samples and $32 \mathrm{NE}$ samples. The raw data can be availably downloaded and analyzed from NCBI-GEO.

2.2 Assessment of immune cell infiltration. The gene expression matrix was uploaded to the online tool named xCell(https://xcell.ucsf.edu/) (14). The "Rooney signature ( $N=7)$ " column was chosen to calculate the proportions of 7 types of infiltration immune cells in EC and NE samples, including B cells, CD 4+T cells, CD8+T cells, DC cells, macrophages, NK cells, neutrophils.

\subsection{Construction of the coexpression network and identification of the hub module related to immune} cells. The gene co-expression networks of EC samples were constructed by the WGCNA package, and the proportion of immune infiltrated cells was input as WGCNA trait data to identify the hub module (15). The 
Pearson test $(P<0.05)$ was used to calculate the correlation between module eigengenes and immune cells, and the module most relevant to immune cells was selected and defined as the hub module.

2.4 Screening of the differentially expressed genes (DEGs). The downloaded matrix was used to screen the differentially expressed genes (DEGs) of EC samples with the limitation of |log2 fold change (FC)|> 1 and an adjusted P-value of $<0.05$ by the limma package. In addition, the differential expression of those hub genes in EC and NE samples were analyzed by the Wilcoxon test and visualized using the ggplot2 package.

2.5 Function enrichment analyses. Search Tool for the Retrieval of Interacting Genes/Proteins (STRING) database (http://string-db.org/) was used to identify the interactions among the genes and Cytoscape 3.8.2 (https://cytoscape.org/) was used to analyze its connectivity degree and visualize the proteinprotein interaction (PPI) network $(19,20)$. Next, the functional analyses including Gene Ontology (GO) terms and Kyoto encyclopedia of genes and genomes (KEGG) pathways, were annotated and visualized using the "clusterProfiler", "org.Hs.eg.db", "topGO", "pathview" and "ggplot2" packages of R (21).

2.6 Identification of immune infiltration related biomarkers for EM. The hub genes were recognized using the Venn diagrams of the overlapping genes identified from the hub module, DEGs and immune related genes (IRGs). IRGs were downloaded from the ImmPort database, which disseminates data to the public for the future of immunology (17).

\section{Results}

3.1 Gene expression signatures of the ectopic lesion of EM. The analysis procedures of this study were shown in Figure 1A. There was a total of 134 endometrial samples and 19746 genes in the GSE141549 profile. Boxplot of this dataset was shown in Figure 1B. A total of 816 DEGs identified from EC and NE samples, consisting of 334 downregulated and 482 upregulated genes (Figure 2A). GO and KEGG analyses of DEGs indicated that complement and coagulation cascades, cytochrome P450 were significantly enriched (Figure 2B-C), which indicated that the dysfunction of the immune system and inflammation is connectively associated with the ectopic lesions of endometriosis.

3.2 Signature of the immune infiltration of EM. 7 types of infiltrating immune cells in the endometrium tissue were estimated by the xCell algorithm and showed in a heatmap and a violin diagram. As shown in the heatmap (Figure $3 \mathrm{~A}$ ), macrophages and neutrophils were the main infiltrating immune cells in the endometrium tissue. In the violin diagram (Figure 3B), the composition of CD4+T cells along with $B$ cells, macrophages and neutrophils were significantly higher in EC samples than NE samples, while NK cells were significantly lower than that of controls. The Pearson test $(p<0.05)$ was then used to evaluate the correlation among 7 types of immune cells (Figure $3 \mathrm{C}$ ). Most of the immune cells in the endometrium tissue were highly positive correlated, which indicated that immune infiltration is so interconnected. For example, neutrophils had the significantly high relationship with macrophages $\left(R^{2}=0.76, p<0.05\right)$ and $B$ cells $\left(R^{2}=0.57, p<0.05\right)$. 
3.3 Construction of WGCNA and Identification of the hub module. The top 30\% variation coefficient of genes (5924 genes) in $102 \mathrm{EC}$ samples and the composition of 7 immune infiltrated cells corresponding to $102 \mathrm{EC}$ samples were input to establish the co-expression networks and identify the hub module related to immune cells using the WGCNA package of R (Figure 4A). When the soft-thresholding power was 8 , a scale-independent topological network was established (Figure 4B). Genes were classified into different modules using the dynamic hybrid cutting method, and the minimum module size cut-off value was 100. A total of nine gene modules were ultimately identified with the dynamic tree cutting method which was enforced for the construction of a hierarchical clustering tree by splitting the dendrogram at relevant transition points and the modules with a difference $<0.25$ were combined (Figure 4C).

To determine the hub module, the correlation between the module and the composition of 7 immune cells was calculated using a Pearson test $(P<0.05)$. In nine modules, the brown module, consisting of 803 genes, was highest related to macrophages $\left(R^{2}=0.89, p=5 e-36\right)$, neutrophils ( $\left.R 2=0.73, p=2 e-18\right), D C$ $(R 2=0.62, p=5 e-12), B$ cells $(R 2=0.47, p=5 e-07)$, and $C D 8+$ cells $(R 2=0.42, p=1 e-05)$ and considered to be the hub module related to immune infiltration (Figure $4 \mathrm{D}$ ).

3.4 Functional analyses of the hub module. The PPI network of the hub module was constructed, and 16 central nodes were selected with the connective degree $>20$ (Figure 5A). Furthermore, the biological functions of the hub module were annotated. According to GO and KEGG analysis, most of the genes were involved in allograft rejection, antigen processing and presentation, cell adhesion molecules, lysosome, phagosome (Figure 5B-C).

3.5 Identification and validation of immune infiltration related biomarkers for EM. There were 1793 IRGs downloaded from the ImmPort database. In the Venn diagram (Figure 6A), there were 7 shared genes (LEP, C3, SLPI, S100A8, TNFSF13B, IL7R, CSF1R) recognized by the results of brown module, DEGs and IRGs mentioned above and put into the string database. Cause the immune response is so interconnected, the hub genes were defined as the genes interacted with each other among 7 shared genes mentioned above, including TNFSF13B, IL7R, CSF1R, LEP (Figure 6B). In Figure 6C, the expression of those hub genes in EC were significantly higher than that of controls. The independent validation profiles GSE7305 and GSE23339 were also downloaded from the GEO database and separately contained 10 paired EC and NE samples, 10 EC and 9 NE samples $(22,23)$. The expression of those 4 hub genes in GSE7305 and GSE23339 was significantly consistent with our result (Supplemental Figure 1AB).

3.6 Identification of clinical characteristics of hub genes. The AUC of those hub genes to distinguish endometriosis patients and normal people were shown in Figure 7A. The AUC of 4 hub genes was higher than 0.8 , with LEP being the maximum 0.906 and CSF1R being the minimum 0.834 . The independent dataset GSE120103 contained 9 paired eutopic endometrium tissue samples of fertile (EM-F) or infertile (EM-IF) women undergoing endometriosis (24). As shown in Figure 7B, the expression of TNFSF13B, IL7R and LEP in EM-IF was significantly higher than that of in EM-F, while CSF1R was much lower. There we 
further inferred those 4 hub genes may be also connectively associated with the common complication infertility of EM.

3.7 Correlation between biomarkers and immune infiltrated cells. The correlation among 4 hub genes (TNFSF13B, IL7R, CSF1R, LEP) and 7 types of immune infiltrated cells (B cells, CD4+T cells, CD8+T cells, DC cells, macrophages, NK cells, neutrophils) was analyzed by the Pearson test and presented in Figure $8 \mathrm{~A}$. The results indicated that hub genes were positively correlated with the composition of immune cells except NK cells. For example (Figure 8B-C), TNFSF13B was positively related to $B$ cells $(R 2=0.60, p<2.2 e-$ $16)$ and CSF1R was positively related to macrophages $(R 2=0.79, p<2.2 e-16)$.

\section{Discussion}

Endometriosis is a common gynecological disease whose origin and pathogenesis are yet unknown. The widely accepted theory is the retrograde menstruation theory, which was proposed by Sampson that menstrual debris could pass through the fallopian tubes and implant into the abdominal cavity in a retrograde way. While retrograde menstruation occurs in about $90 \%$ of menstruating women, the prevalence of endometriosis is only about 6 to $10 \%$ of women of reproductive age $(3,25)$. It indicated that retrograde menstruation theory does not adequately explain the pathogenesis of this disease. Therefore, there must be other possible explanations for it.

A well-functioning immune system could eliminate ectopic endometrial cells from the peritoneal cavity. New findings on the genetics, immunological dysfunction, intrinsic abnormalities, and secreted products of endometriotic lesions are associated with the reduced clearance of endometrial fragments and could facilitate the persistence of endometriosis (25).

Through comprehensive bioinformatic analyses of common dataset GSE141549, we compared the immune infiltration between EC and NE samples by XCell algorithm. The results showed that macrophages and neutrophils were the main infiltrating immune cells in the endometrium tissue, and the composition of $\mathrm{CD} 4+\mathrm{T}$ cells along with $\mathrm{B}$ cells, macrophages and neutrophils were significantly higher in EC samples than NE samples, while NK cells were significantly lower than that of controls. The gene coexpression networks were constructed through WGCNA package, and the composition of immune infiltrated cells was selected as WGCNA trait data. A total of nine gene modules were ultimately identified with the dynamic tree cutting method. The brown module, consisting of 803 genes, was the highest related to most of immune cells like macrophages and neutrophils. A total of 816 DEGs, consisting of 334 downregulated and 482 upregulated genes, were identified from EC and NE samples with the limitation of $\mid \log 2$ fold change (FC) $\mid>1$ and an adjusted P-value of $<0.05$ by the limma package. The GO and KEGG functional analysis of DEGs indicated that the dysfunction of the immune system and inflammation is connectively associated with the ectopic lesions of endometriosis. The brown module, DEGs and IRGs mentioned above were put into the Venn diagram and STRING database. The overlapping genes interacted with each other were defined as the hub genes related to immune infiltrated of EM, 
including TNFSF13B, IL7R, CSF1R and LEP. And those hub genes were positively correlated with the composition of most immune cells.

The expression of those hub genes in EC were significantly higher than that of controls. And the AUC of 4 hub genes to distinguish endometriosis patients and normal people were all higher than 0.8 , with LEP being the maximum 0.906 and CSF1R being the minimum 0.834 . The independent datasets GSE7305 and GSE23339 were used to validate the hub genes, and the results were completely consistent with our results mentioned above. In independent dataset GSE120103, the expression of TNFSF13B, IL7R and LEP in EM-IF was significantly higher than that of in EM-F, while CSF1R was much lower. There we further inferred those 4 hub genes were also connectively associated with the common complication infertility of EM.

The protein encoded by TNFSF13B is a cytokine that belongs to the tumor necrosis factor (TNF) ligand family. This cytokine, acts as a potent B lymphocyte stimulator (BlyS), is produced by macrophages, and necessary for normal B cell differentiation and proliferation (26). Notably, high levels of BlyS overstimulate various $B$ cell responses, leading to other conditions such as autoimmune diseases, allergic diseases, infections and malignancies (27). There was also indicated that the $-817 \mathrm{C} / \mathrm{T}$ in the promoter region of this gene possibly associated with idiopathic infertility in Brazilian population (28).

The protein encoded by IL7R is a receptor for interleukin 7 (IL7). IL7 and IL7R are crucial for innate lymphoid cell development and maintenance and are also implicated in autoimmune and chronic inflammatory diseases, as well as in cancer (29).There are also emerging reports of IL7R signaling contributing to the progression of lymphoid malignancies such as $T$ cell acute lymphoblastic leukemia and its effective anti-IL7R targeting antibody therapies (30). However, the role of IL7R in endometriosis disease remains unexplored.

The protein encoded by CSF1R is the receptor for colony stimulating factor 1 (CSF1), a cytokine which controls the production, differentiation, and function of macrophages. This receptor mediates most of the biological effects of this cytokine. CSF1 interaction with CSF1R has been implicated in the growth, invasion, and metastasis of several types of cancer, including breast and endometrial cancers $(31,32)$. In our study, the expression of CSF1 was significantly higher in EC than NE samples. Increased CSF1R levels has been implicated in the pathogenesis of endometriosis. It is also suggested that endometrial tissue involved in lesion formation is highly responsive to CSF-1 signaling $(33,34)$.

Leptin (LEP) encodes a protein that is secreted by white adipocytes into the circulation and plays a major role in the regulation of energy homeostasis. Apart from its metabolic properties, leptin is also involved in the regulation of immune and inflammatory responses, hematopoiesis, angiogenesis, and reproduction (35-37). Increased levels of leptin have been identified in peritoneal fluid from primary infertility women with endometriosis. In addition, the correlation between leptin levels and the endometriosis stage are significantly positive $(38,39)$. 
In a summary, our study indicated that the immune infiltration was highly associated with the the ectopic lesions of EM. And the immune related biomarkers (TNFSF13B, IL7R, CSF1R, LEP) may be helpful in the development of the pathogenesis, diagnosis and even the potential therapeutic targets of EM. However, there is still a certain degree of limitation existing in our study. To the best of our knowledge, further investigations of these hub genes are needed to identify the molecular mechanism and to validate their immunotherapy effect on EM.

\section{Conclusions}

In the present study, it was showed that macrophages and neutrophils were the main infiltrating immune cells in the endometrium tissue and 4 biomarkers related to immune infiltration in ectopic endometria of EM patients were identified using WGCNA and xCell algorithm, including TNFSF13B, IL7R, CSF1R and LEP. In the independent validated datasets, the expression of those hub genes was upregulated and consistent with our results. The area under the ROC curve (AUC) of 4 hub genes to distinguish EM and normal people were all higher than 0.8 . And the expression of 4 hub genes was significantly concerned with the common complication infertility of EM.

\section{List Of Abbreviations}

EM: endometriosis; GEO: the gene expression omnibus database; WGCNA: the weighted gene coexpression network; DEGs: the differentially expressed genes; AUC: the area under the ROC curves; EC: ectopic endometria; ImmPort: the Immunology Database and Analysis Portal; NE: normal endometria; STRING: Search Tool for the Retrieval of Interacting Genes/Proteins database; PPI: protein-protein interaction network; GO: functional analyses including Gene Ontology terms; KEGG: Kyoto encyclopedia of genes and genomes pathways; IRGs: the immune related genes; EM-F: fertile people undergoing endometriosis; EM-IF: infertile people undergoing endometriosis; TNFSF13B: tumor necrosis factor superfamily, member 13b; IL7R: interleukin 7 receptor; CSF1R: colony stimulating factor 1 receptor; LEP: leptin.

\section{Declarations}

Ethics approval and consent to participate: Not appliable.

Consent for publication: All authors have agreed to the publication of the work.

Availability of data and materials: Data used or analyzed during the current study are available from the corresponding author on reasonable request. The profiles analyzed in this study were downloaded from the online repositories.

Competing interests: there is no conflict of interest that I should disclose.

Funding: Not appliable. 
Author Contributions: Ke Zhang designed the experimental apparatus, discussed the results and implications, and commented on the manuscript at all stages. Xiao xie and Lihao Zou performed the experiments. The research direction was provided by Suiqun Guo.

Acknowledgements: The authors would like to thank the support offered by every member of the department of Obstetrics and Gynecology in the Third Affiliated Hospital, Southern Medical University.

\section{References}

1. Giudice LC. Clinical practice. Endometriosis. N Engl J Med. 2010;362(25):2389-98.

2. Rolla E. Endometriosis: advances and controversies in classification, pathogenesis, diagnosis, and treatment. F1000Research. 2019;8.

3. Mehedintu C, Plotogea M, lonescu S, Antonovici M. Endometriosis still a challenge. Journal of medicine and life. 2014;7(3):349-57.

4. Nnoaham K, Hummelshoj L, Webster P, d'Hooghe T, de Cicco Nardone F, de Cicco Nardone C, et al. Impact of endometriosis on quality of life and work productivity: a multicenter study across ten countries. Fertility and sterility. 2011;96(2):366-73.e8.

5. Nowak N, Fischer O, Gust T, Fuhrmann U, Habenicht U, Schmidt A. Intraperitoneal inflammation decreases endometriosis in a mouse model. Human reproduction (Oxford, England). 2008;23(11):2466-74.

6. Berbic M, Fraser IS. Immunology of normal and abnormal menstruation. Womens Health (Lond). 2013;9(4):387-95.

7. Olkowska-Truchanowicz J, Bocian K, Maksym R, Białoszewska A, Włodarczyk D, Baranowski W, et al. CD 4 $\triangle C D 25 \rrbracket F O X P 3 \rrbracket$ regulatory $T$ cells in peripheral blood and peritoneal fluid of patients with endometriosis. Human reproduction (Oxford, England). 2013;28(1):119-24.

8. Bellelis P, Barbeiro DF, Rizzo LV, Baracat EC, Abrão MS, Podgaec S. Transcriptional changes in the expression of chemokines related to natural killer and T-regulatory cells in patients with deep infiltrative endometriosis. Fertil Steril. 2013;99(7):1987-93.

9. Monsanto SP, Edwards AK, Zhou J, Nagarkatti P, Nagarkatti M, Young SL, et al. Surgical removal of endometriotic lesions alters local and systemic proinflammatory cytokines in endometriosis patients. Fertil Steril. 2016;105(4):968-77.e5.

10. Kobayashi $\mathrm{H}$, Higashiura Y, Shigetomi H, Kajihara H. Pathogenesis of endometriosis: the role of initial infection and subsequent sterile inflammation (Review). Mol Med Rep. 2014;9(1):9-15.

11. Miller JE, Ahn SH, Monsanto SP, Khalaj K, Koti M, Tayade C. Implications of immune dysfunction on endometriosis associated infertility. Oncotarget. 2017;8(4):7138-47.

12. Fachal L, Aschard H, Beesley J, Barnes D, Allen J, Kar S, et al. Fine-mapping of 150 breast cancer risk regions identifies 191 likely target genes. Nature genetics. 2020;52(1):56-73. 
13. Dehghan A. Genome-Wide Association Studies. Methods in molecular biology (Clifton, NJ). 2018;1793:37-49.

14. Aran D, Hu Z, Butte A. xCell: digitally portraying the tissue cellular heterogeneity landscape. Genome biology. 2017;18(1):220.

15. Luo Y, Coskun V, Liang A, Yu J, Cheng L, Ge W, et al. Single-cell transcriptome analyses reveal signals to activate dormant neural stem cells. Cell. 2015;161(5):1175-86.

16. Bhattacharya S, Dunn P, Thomas CG, Smith B, Schaefer H, Chen J, et al. ImmPort, toward repurposing of open access immunological assay data for translational and clinical research. Sci Data. 2018;5:180015.

17. Bhattacharya S, Andorf S, Gomes L, Dunn P, Schaefer H, Pontius J, et al. ImmPort: disseminating data to the public for the future of immunology. Immunol Res. 2014;58(2-3):234-9.

18. Gabriel M, Fey V, Heinosalo T, Adhikari P, Rytkönen K, Komulainen T, et al. A relational database to identify differentially expressed genes in the endometrium and endometriosis lesions. Sci Data. 2020;7(1):284.

19. Szklarczyk D, Gable A, Nastou K, Lyon D, Kirsch R, Pyysalo S, et al. Correction to 'The STRING database in 2021: customizable protein-protein networks, and functional characterization of useruploaded gene/measurement sets'. Nucleic acids research. 2021.

20. Otasek D, Morris J, Bouças J, Pico A, Demchak B. Cytoscape Automation: empowering workflowbased network analysis. Genome biology. 2019;20(1):185.

21. Kanehisa M, Goto S, Sato Y, Kawashima M, Furumichi M, Tanabe M. Data, information, knowledge and principle: back to metabolism in KEGG. Nucleic acids research. 2014;42:D199-205.

22. Hever A, Roth R, Hevezi P, Marin M, Acosta J, Acosta $H$, et al. Human endometriosis is associated with plasma cells and overexpression of $B$ lymphocyte stimulator. Proceedings of the National Academy of Sciences of the United States of America. 2007;104(30):12451-6.

23. Hawkins SM, Creighton CJ, Han DY, Zariff A, Anderson ML, Gunaratne PH, et al. Functional microRNA involved in endometriosis. Mol Endocrinol. 2011;25(5):821-32.

24. Bhat MA, Sharma JB, Roy KK, Sengupta J, Ghosh D. Genomic evidence of $Y$ chromosome microchimerism in the endometrium during endometriosis and in cases of infertility. Reprod Biol Endocrinol. 2019;17(1):22.

25. Berveiller P, Ropert S, Mir O. Endometriosis. The New England journal of medicine. 2020;383(2):193-4.

26. Schiemann B, Gommerman JL, Vora K, Cachero TG, Shulga-Morskaya S, Dobles M, et al. An essential role for BAFF in the normal development of B cells through a BCMA-independent pathway. Science. 2001;293(5537):2111-4.

27. Lied GA, Berstad A. Functional and clinical aspects of the B-cell-activating factor (BAFF): a narrative review. Scand J Immunol. 2011;73(1):1-7.

28. Christofolini DM, Cavalheiro CM, Teles JS, Lerner TG, Brandes A, Bianco B, et al. Promoter -817C>T variant of B lymphocyte stimulator gene (BLyS) and susceptibility to endometriosis-related infertility 
and idiopathic infertility in Brazilian population. Scand J Immunol. 2011;74(6):628-31.

29. Barata JT, Durum SK, Seddon B. Flip the coin: IL-7 and IL-7R in health and disease. Nat Immunol. 2019;20(12):1584-93.

30. Yasunaga M. Antibody therapeutics and immunoregulation in cancer and autoimmune disease. Semin Cancer Biol. 2020;64:1-12.

31. Cassetta L, Fragkogianni S, Sims AH, Swierczak A, Forrester LM, Zhang H, et al. Human TumorAssociated Macrophage and Monocyte Transcriptional Landscapes Reveal Cancer-Specific Reprogramming, Biomarkers, and Therapeutic Targets. Cancer Cell. 2019;35(4):588-602.e10.

32. Hua F, Tian Y, Gao Y, Li C, Liu X. Colony-stimulating factor 1 receptor inhibition blocks macrophage infiltration and endometrial cancer cell proliferation. Mol Med Rep. 2019;19(4):3139-47.

33. Jensen JR, Witz CA, Schenken RS, Tekmal RR. A potential role for colony-stimulating factor 1 in the genesis of the early endometriotic lesion. Fertil Steril. 2010;93(1):251-6.

34. Budrys NM, Nair HB, Liu YG, Kirma NB, Binkley PA, Kumar S, et al. Increased expression of macrophage colony-stimulating factor and its receptor in patients with endometriosis. Fertil Steril. 2012;97(5):1129-35.e1.

35. Gonçalves HF, Zendron C, Cavalcante FS, Aiceles V, Oliveira MA, Manaia JH, et al. Leptin, its receptor and aromatase expression in deep infiltrating endometriosis. J Ovarian Res. 2015;8:53.

36. Nácul AP, Lecke SB, Edelweiss MI, Morsch DM, Spritzer PM. Gene expression of leptin and long leptin receptor isoform in endometriosis: a case-control study. Obstet Gynecol Int. 2013;2013:879618.

37. DePaoli AM. 20 years of leptin: leptin in common obesity and associated disorders of metabolism. J Endocrinol. 2014;223(1):T71-81.

38. Matarese G, Alviggi C, Sanna V, Howard JK, Lord GM, Carravetta C, et al. Increased leptin levels in serum and peritoneal fluid of patients with pelvic endometriosis. J Clin Endocrinol Metab. 2000;85(7):2483-7.

39. Gungor T, Kanat-Pektas M, Karayalcin R, Mollamahmutoglu L. Peritoneal fluid and serum leptin concentrations in women with primary infertility. Arch Gynecol Obstet. 2009;279(3):361-4.

\section{Figures}




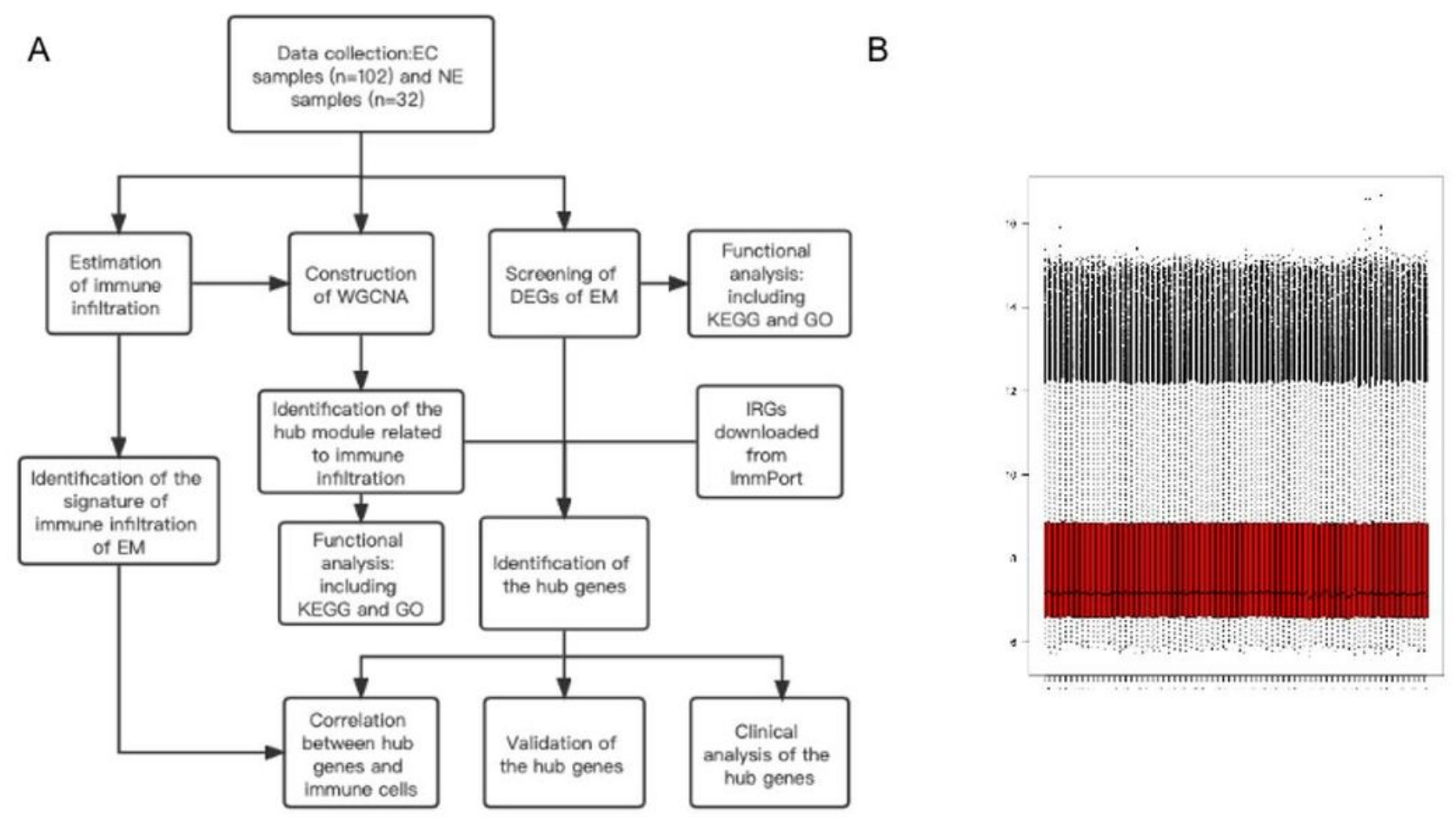

Figure 1. The analysis procedures of this study and the gene expression of GSE141549. (A) The analysis procedures. (B) Boxplot showed the gene expression has been initially corrected to move the batch effects and combined.

\section{Figure 1}

See image above for figure legend 
A

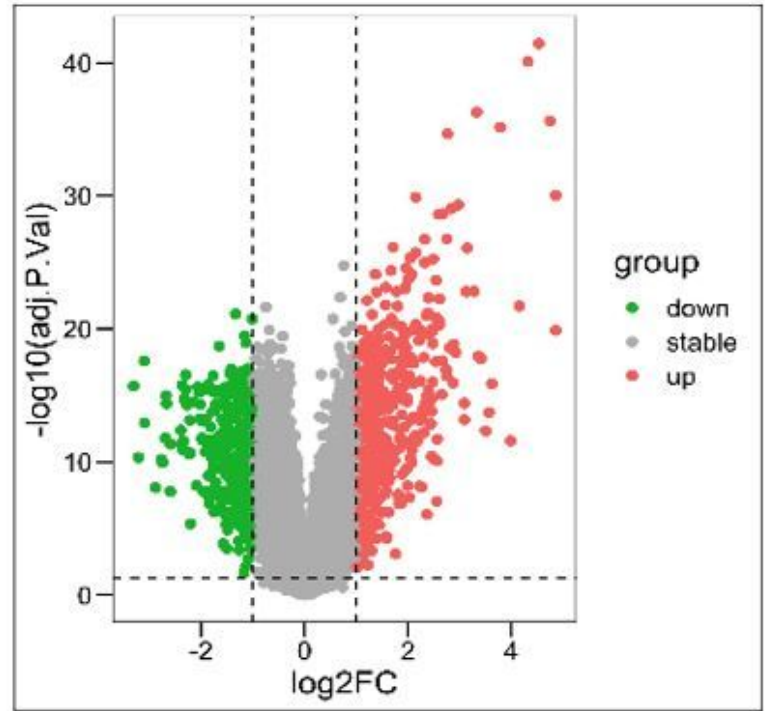

Figure 2. Gene expression signatures of the ectopic lesion of EM. (A) The volcano plot of the DEGs in GSE141549. The green dots represented downregulated genes, the red dots represented the upregulated genes, and the gray dots represented the stable genes.

B

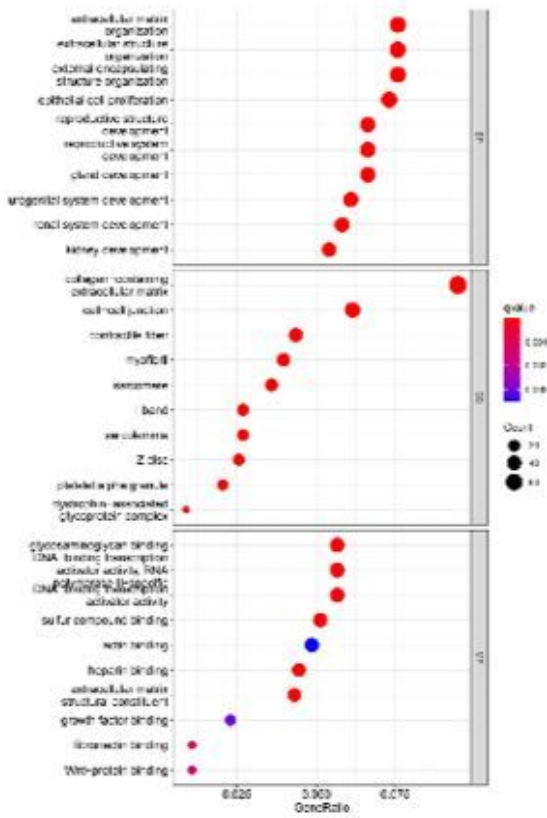

C

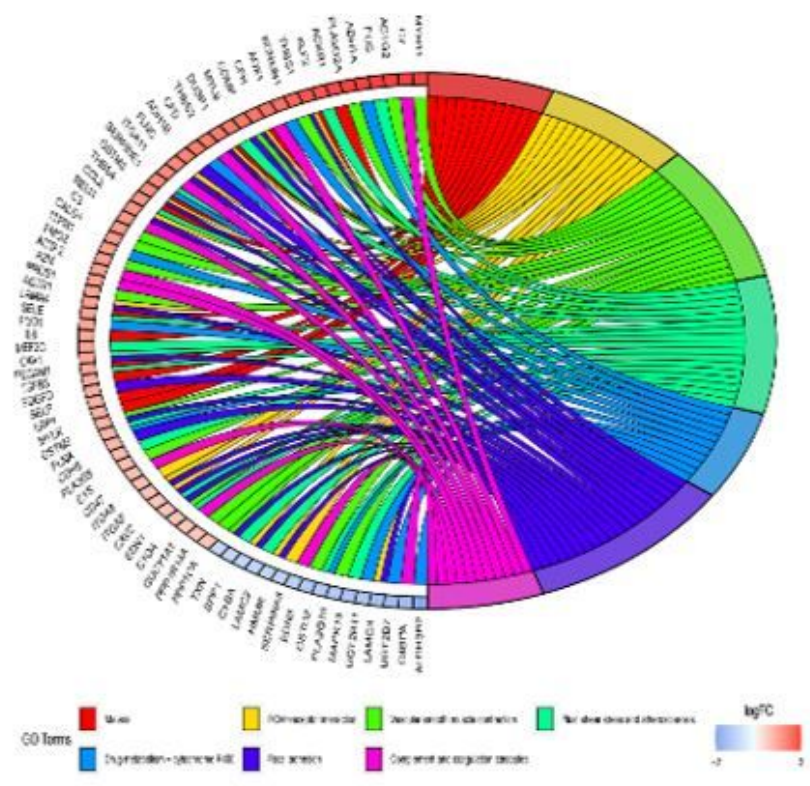

Figure 2. Gene expression signatures of the ectopic lesion of EM. (B) GO enrichment analysis of DEGs. Dot sizes reflected the number of genes associated with relative pathways, and dot colors indicated the qvalues.(C) The KEGG enrichment analysis of DEGs showed the top 7 pathways.

Figure 2

See image above for figure legend 
A

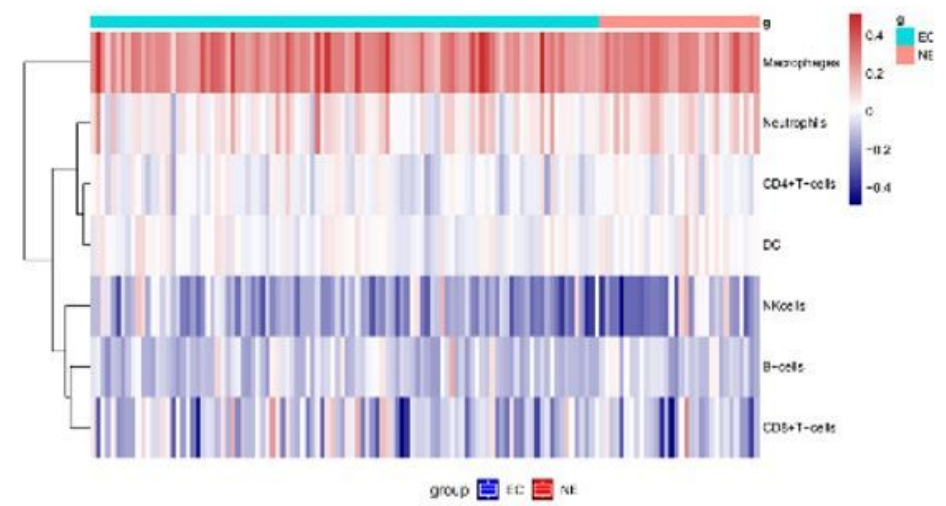

B

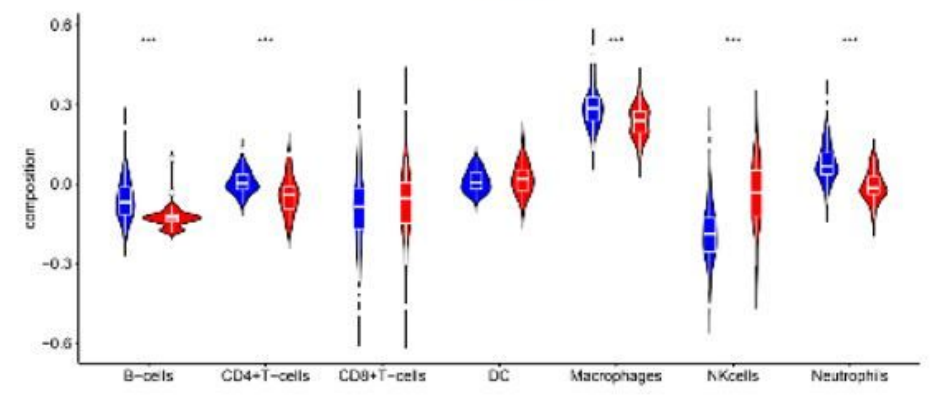

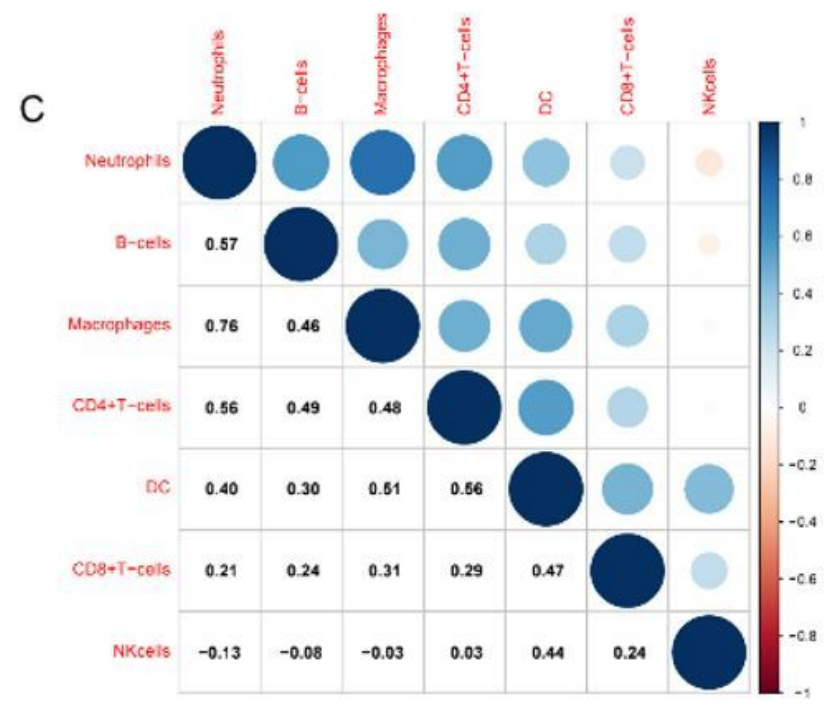

Figure 3. Estimation of infiltrating immune cells. (A) Heatmap and (B) violin diagram of 7 types of infiltrating immune cells in the endometrium tissue. (C) The correlation among 7 types of immune cells. Data was compared with the Wilcoxon test. ${ }^{*} \mathrm{P}<0.05$, ${ }^{* \star} \mathrm{P}<0.01$, and ${ }^{\star \star *} \mathrm{P}<0.001$. ns, no significance.

Figure 3

See image above for figure legend 
A

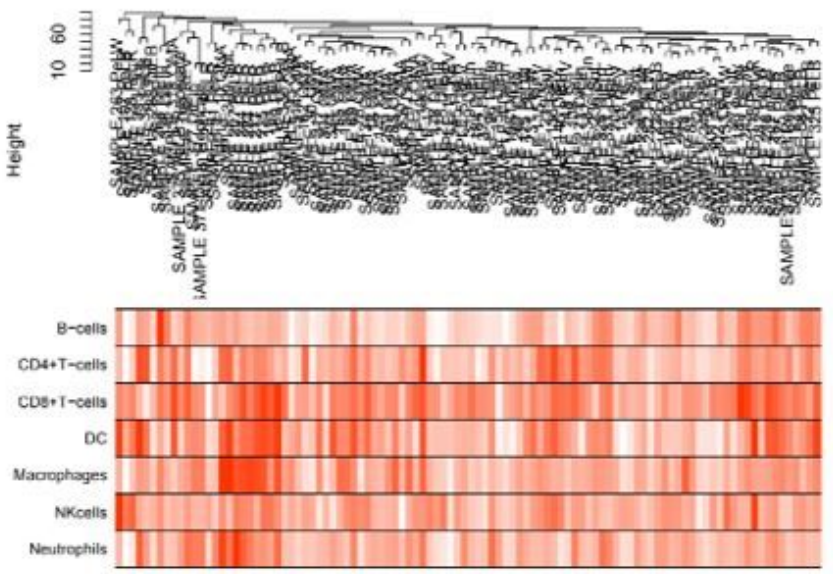

B

Scale independence

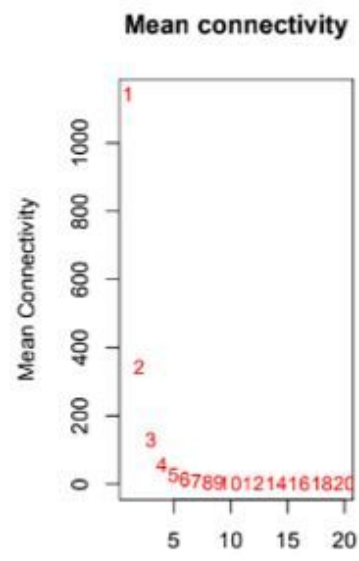

Soft Threshold (power)

Figure 4. Cluster analysis and construction of WGCNA. (A) Sample dendrogram and trait heatmap. In the heatmap, the darker the color, the higher the composition of immune cells. (B) The scale-free fitting index of different soft threshold power $(\beta)$ and the average connectivity of various soft threshold powers.
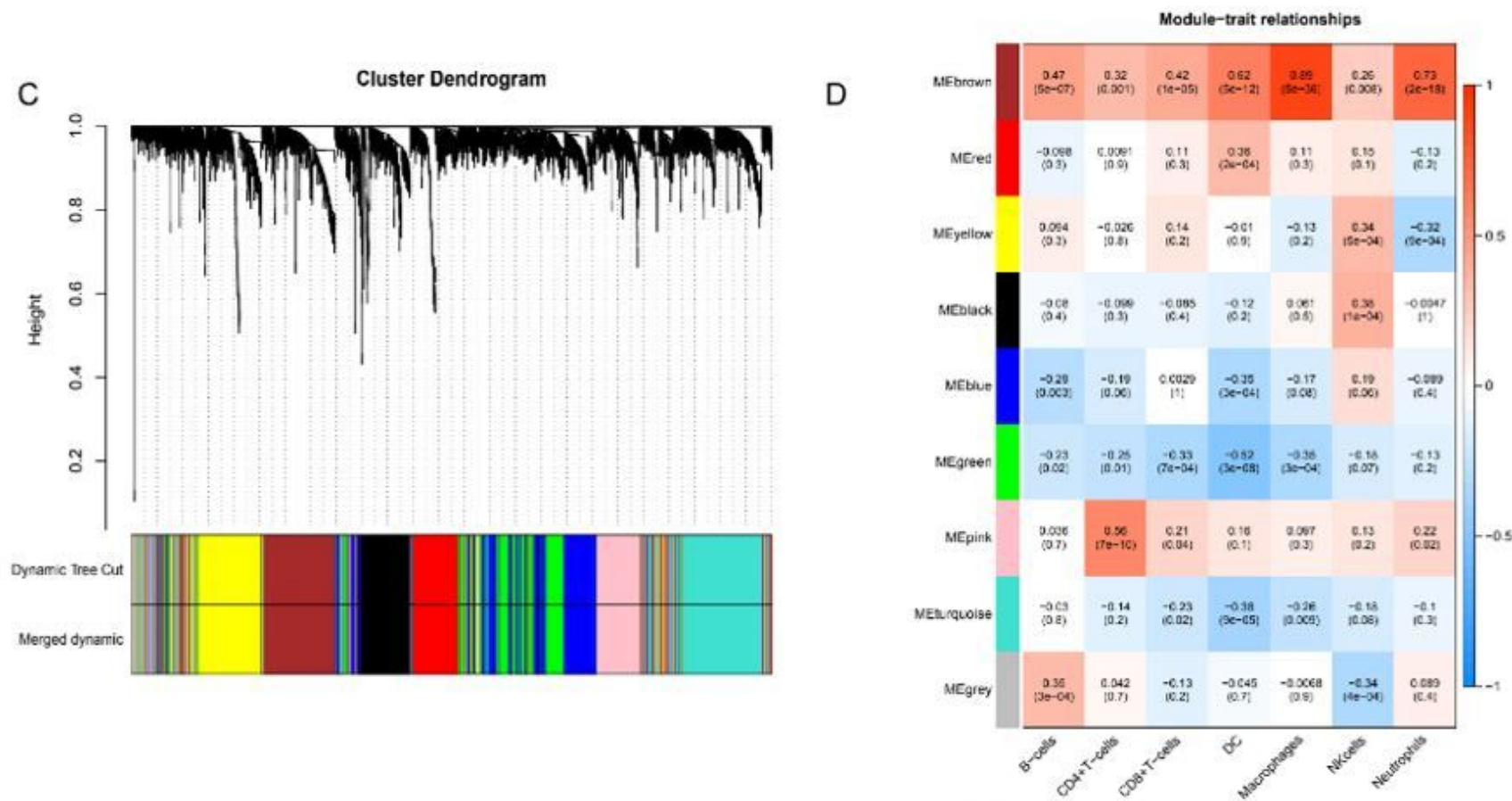

Figure 4. Cluster analysis and construction of WGCNA. (C) Clustering results of modules in gene data in WGCNA analysis. (D) Heatmaps of the correlation between the module characteristic genes and immune cells.

\section{Figure 4}

See image above for figure legend 
A
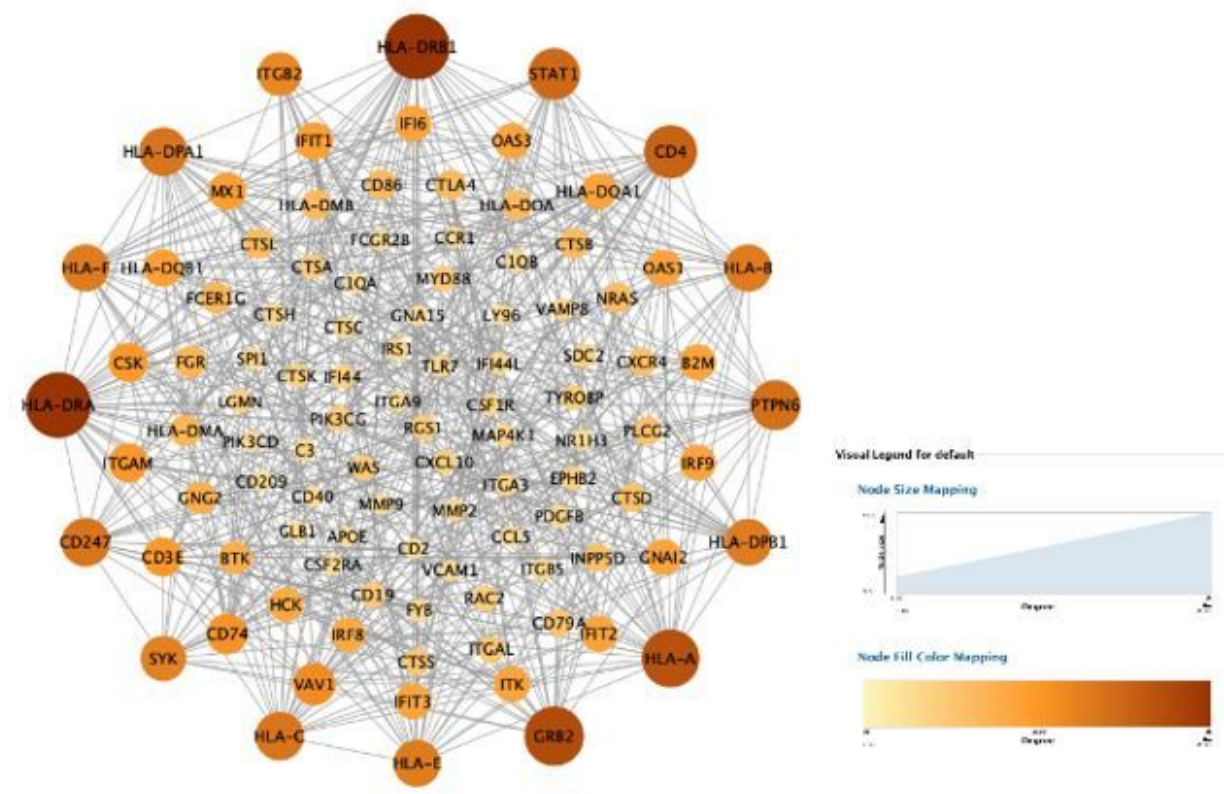

Figure 5. Analysis of the hub module. (A) The PPI network of the hub module. Nodes represented genes in the hub module, and edges represented interactions. The node presented with a connective degree $>10$.

B

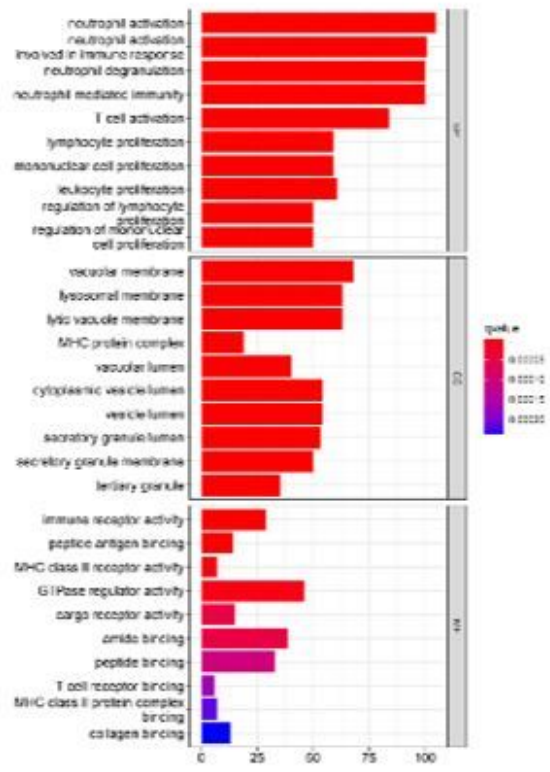

C

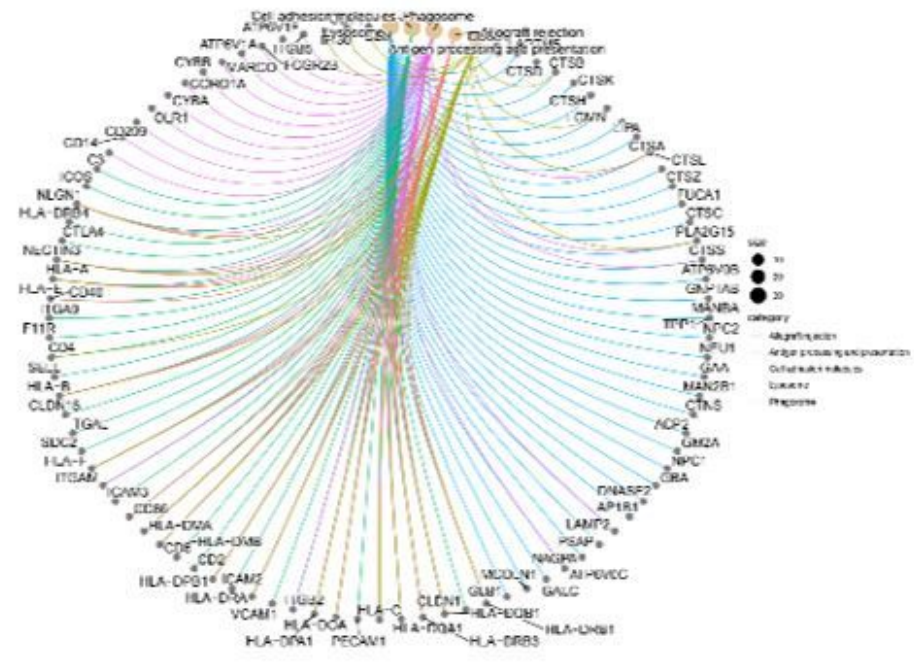

Figure 5. Analysis of the hub module. (B) GO enrichment analysis of the hub module. Dot sizes reflected the number of genes associated with relative pathways, and dot colors indicated the qvalues.(C) The KEGG enrichment analysis of the hub module showed the top 5 pathways.

\section{Figure 5}

See image above for figure legend 
A

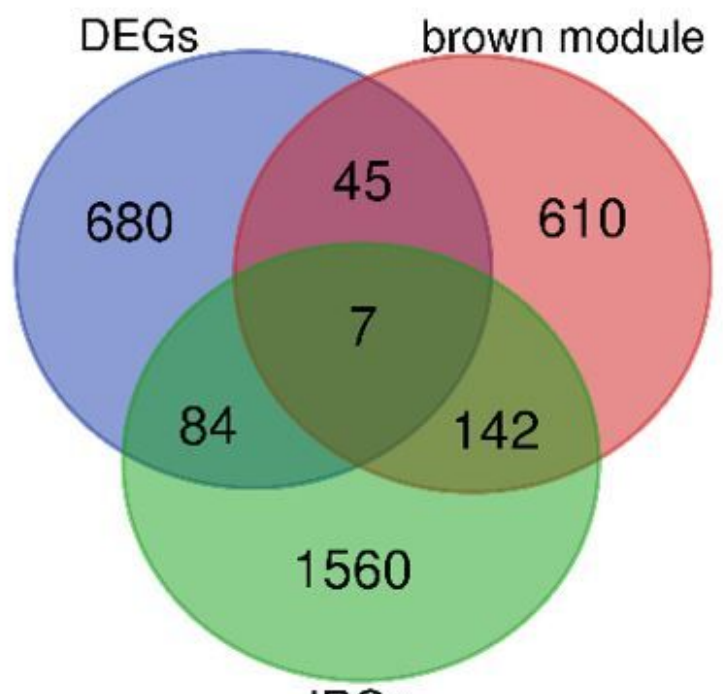

IRGs

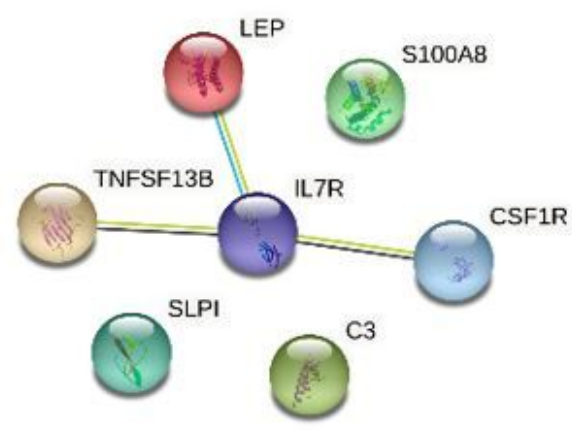

Figure 6. Identification and validation of hub genes. (A) Venn diagram of the DEGs, IRGs and the hub module. (B)The PPI of the shared genes. The genes interacted with each other were defined as the hub genes.

C

group 官 EC 国 NE

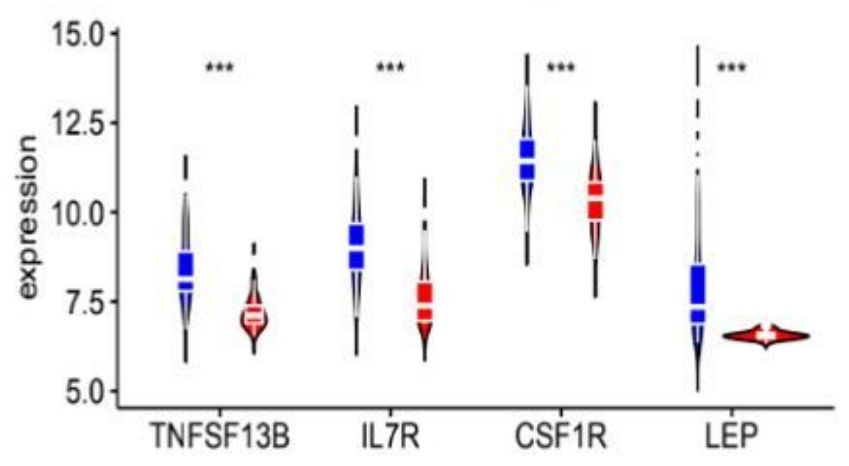

Figure 6. Identification and validation of hub genes. (C) Violin diagram of 4 hub genes in EC and NE samples in GSE141549. Data was compared with the Wilcoxon test. ${ }^{\star} P<0.05$, ${ }^{\star \star} P<$ 0.01 , and ${ }^{\star \star \star} P<0.001$. ns, no significance.

\section{Figure 6}

See image above for figure legend 
A

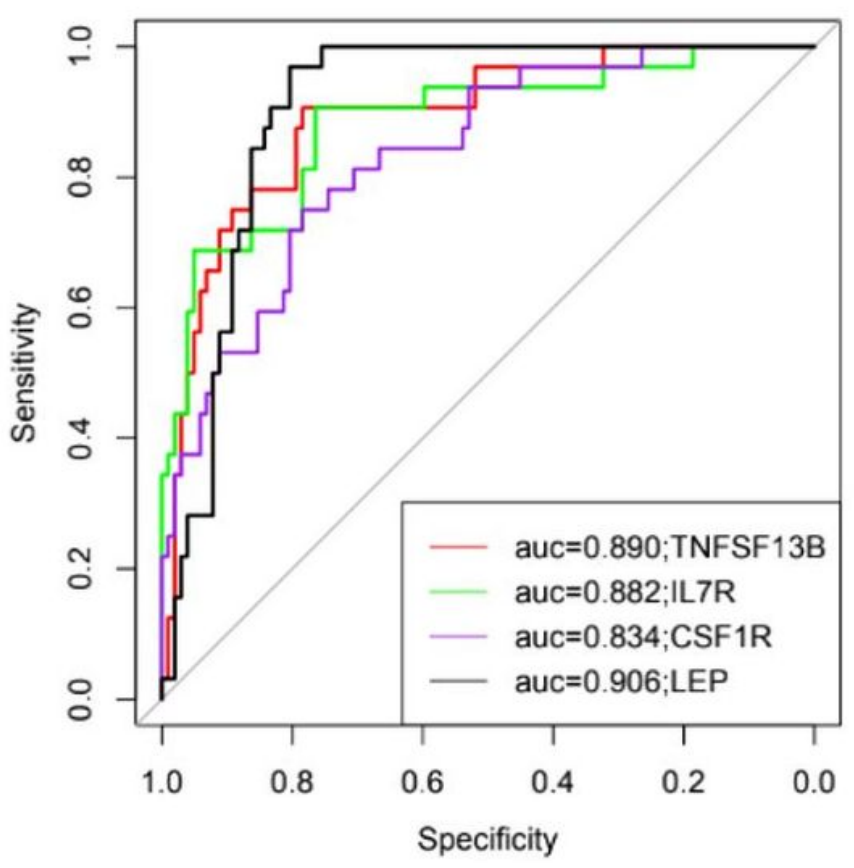

B

group 审 EM-F

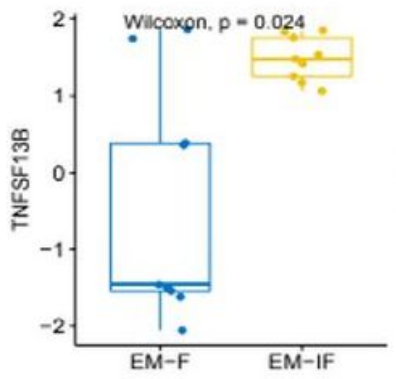

group 审 $\mathrm{FM}-\mathrm{F}$ 运 $\mathrm{EM}-\mathrm{FF}$

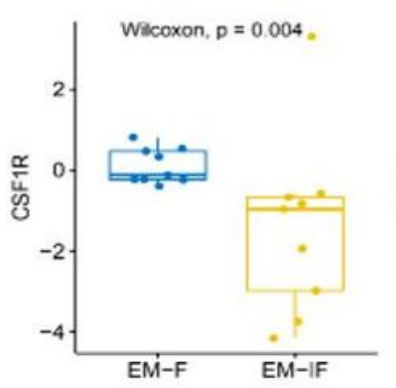

group 安 EM-F

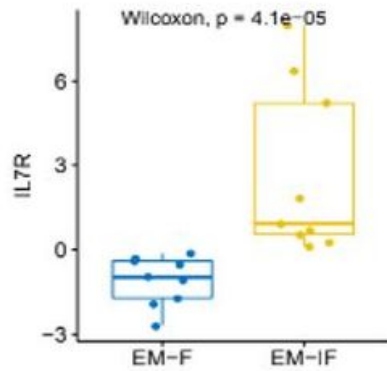

group 审 EM-F E⿱㇒日勺 $\mathrm{EM}-\mathrm{F}$

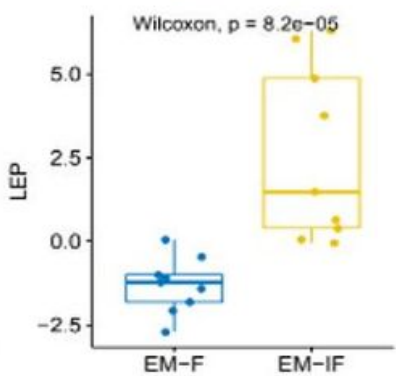

Figure 7. Clinical characteristics of hub genes. (A) ROC curves for 4 hub genes. (B) Boxplot of 4 hub gene in EM-IF and EM-F samples of GSE120103. Data was compared with the Wilcoxon test.

Figure 7

See image above for figure legend 


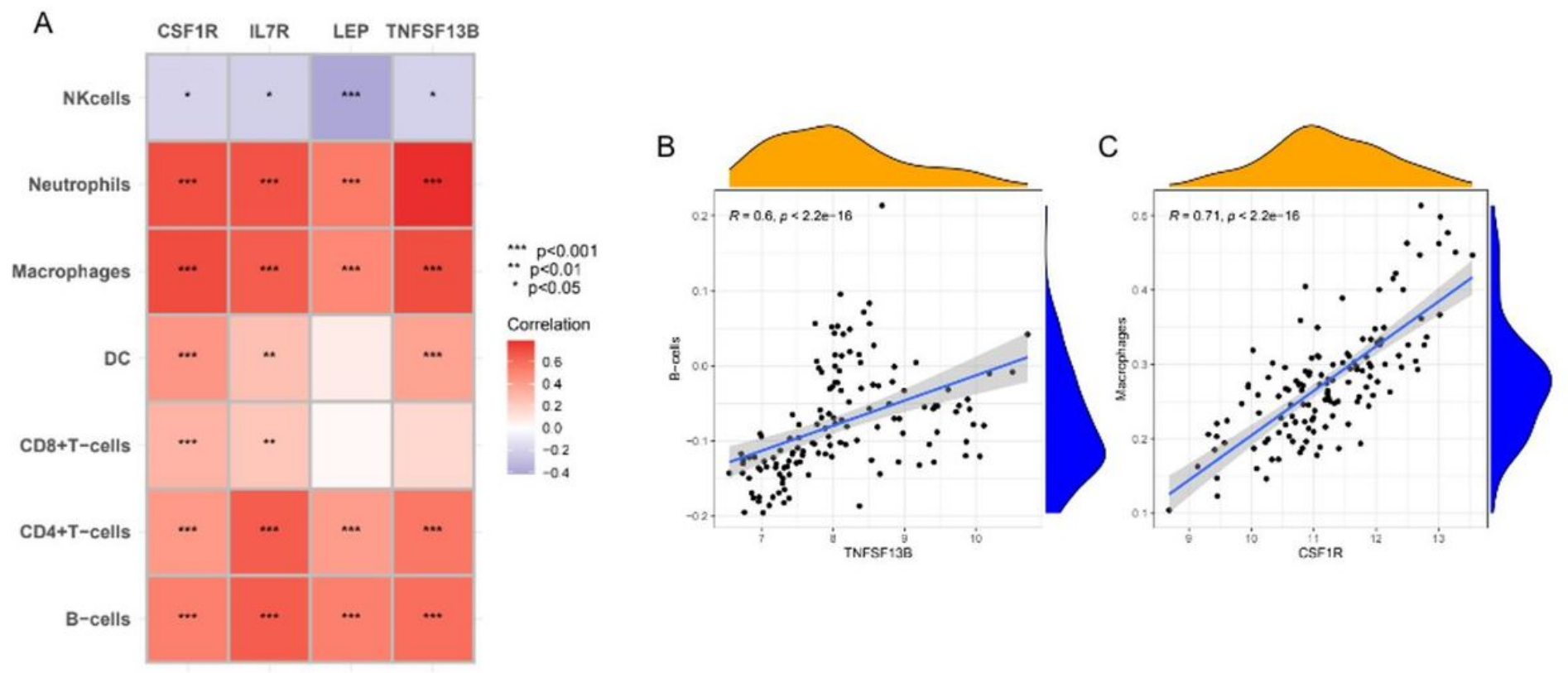

Figure 8. Correlation between biomarkers and immune cells. (A) The correlation between 4 hub genes and 7 immune cells. (B) Scatter diagram of the correlation between TNFSF13B and $B$ cells. (C) Scatter diagram of the correlation between CSF1R and macrophages.

\section{Figure 8}

See image above for figure legend

\section{Supplementary Files}

This is a list of supplementary files associated with this preprint. Click to download.

- SuppFig1.jpg 